\title{
The evolved early-type binary HDE 228766 revisited ${ }^{\star}$
}

\author{
G. Rauw $^{1}$, P. A. Crowther ${ }^{2}$, P. R. J. Eenens ${ }^{3}$, J. Manfroid ${ }^{1, \star \star}$, and J.-M. Vreux ${ }^{1}$ \\ 1 Institut d'Astrophysique et de Géophysique, Université de Liège, Allée du 6 Août, Bât. B5c, \\ 4000 Liège (Sart Tilman), Belgium \\ 2 Department of Physics \& Astronomy, University College London, Gower Street, London WC1E 6BT, UK \\ 3 Departemento de Astronomia, Universidad de Guanajuato, Apartado 144, 36000 Guanajuato Gto, Mexico
}

Received 6 May 2002 / Accepted 18 June 2002

\begin{abstract}
We use an extensive set of spectroscopic observations to reinvestigate the properties of the massive binary HDE 228766. Conventional classification criteria suggest that HDE 228766 consists of an O7 primary and an $\mathrm{Of}^{+}$secondary. However, several spectral features of the secondary, such as the simultaneous presence of $\mathrm{N}$ III, $\mathrm{N}$ IV and $\mathrm{N}$ v emissions, make it a rather unusual object. We find that the orbital motion of the secondary is probably best described by the radial velocities of the narrow $\mathrm{N}$ III emission lines. Our orbital solution yields $m_{1} \sin ^{3} i=31.7$ and $m_{2} \sin ^{3} i=25.5 M_{\odot}$ for the primary and secondary respectively. The He II $\lambda 4542$ absorption in the secondary's spectrum appears considerably blue-shifted with respect to the narrow emission lines, indicating that the absorption is probably formed in the accelerating part of the secondary's wind. We use a tomographic technique to investigate the profile variability of the broader emission lines. In addition to a strong emission from the secondary, the $\mathrm{H} \alpha$ line displays a weak emission feature that is probably associated with a wind interaction region located near the surface of the primary star. Finally, our analysis of the spectrum with a non-LTE code indicates that the secondary is an evolved object that exhibits some CNO processed material in its atmosphere and has a large mass loss rate. Assuming a distance of $3.5 \mathrm{kpc}$ (which follows from adopting $M_{V, \mathrm{~s}}=-6$ for the secondary) we infer $\dot{M} \simeq 10^{-5} M_{\odot} \mathrm{yr}^{-1}$ for the secondary. Our results suggest that HDE 228766 could be in a post-Roche lobe overflow stage. The secondary may be classified as WN8ha and is currently in a transition stage between a "normal" Of star and a WNL-type Wolf-Rayet star.
\end{abstract}

Key words. binaries: spectroscopic - stars: early-type - stars: fundamental parameters - stars: individual: HDE 228766

\section{Introduction}

HDE 228766 (=BD $\left.+36^{\circ} 3991\right)$ was first reported to be a spectroscopic binary with a period of about $10.6 \mathrm{~d}$ by Hiltner (1951). In addition to absorption lines of $\mathrm{HI}$, He I and He II, the spectrograms collected by Hiltner revealed the presence of emission lines of He II $\lambda 4686$ and N IV $\lambda 4058$ and this author classified HDE 228766 thus as "WN7 or slightly earlier". The Wolf-Rayet classification of this system was soon questioned by several authors. In fact, Roman (1951) noted that the emission lines in the spectrum of HDE 228766 were rather narrow, unlike what was observed for typical Wolf-Rayet stars, and therefore Roman suggested that HDE 228766 should be called an Of star. Hiltner \& Schild (1966) reclassified HDE 228766 as an Of-type system and this result was also supported by Walborn (1973) who suggested that the system consists of an early $\mathrm{Of}$ star and a rapidely rotating late $\mathrm{O}$ supergiant. Very recently, Walborn et al. (2002) classified the combined spectrum of HDE 228766 as O3.5 If*.

Send offprint requests to: G. Rauw, e-mail: rauw@astro.ulg. ac . be

* Based on observations collected at the Observatoire de Haute Provence (France) and the Observatorio Astronómico Nacional of San Pedro Mártir (Mexico).

$\star \star$ Directeur de Recherches FNRS (Belgium).
A leap forward in the study of this system came with the work of Massey \& Conti (1977, hereafter MC77). These authors derived an $\mathrm{O} 7.5+\mathrm{O} 5.5 \mathrm{f}$ classification for the primary and secondary respectively. More importantly, they succeeded for the first time to resolve some of the absorption lines in the spectrum of HDE 228766 into two components. In fact, earlier investigators (Hiltner 1951; Hart 1957) failed to resolve these lines and assigned the entire absorption spectrum to the late O primary star. The older orbital solutions hence only provided upper limits on the true orbital motion of the late O component. MC77 found the apparent systemic velocity of the secondary's absorptions to be considerably blue-shifted relative to the primary, while the He II $\lambda 4686$ emission line appeared red-shifted.

Beside its interest for our understanding of stellar evolution, HDE 228766 could also be considered a promising target for studies of stellar wind interactions. The fact that this binary was quoted as a moderately bright X-ray source (Chlebowski et al. 1989) makes it indeed a good candidate for a colliding wind phenomenon. These considerations prompted us to reinvestigate the properties of this binary system with a particular emphasis on the search for phase-locked emission line profile variability that is often associated with a wind-wind collision.

In this paper, we present an extensive set of new spectroscopic observations of HDE 228766. In Sect. 3, we discuss 
the spectral features of HDE 228766. Radial velocity measurements are used in Sect. 4 to derive new orbital solutions. The emission line profile variability is analysed in Sect. 5. Finally, a discussion of the fundamental parameters of this system and of the wind interaction phenomenon is presented in Sect. 6 .

\section{Observations and data reduction}

The bulk of our observations were collected during several observing campaigns at the Observatoire de Haute-Provence (OHP). We used the Carelec and Aurélie spectrographs respectively attached to the 1.93 and $1.52 \mathrm{~m}$ telescopes at OHP. Table 1 provides a summary of the various spectral ranges covered and the corresponding reciprocal dispersions.

For the Carelec observations until 1990, we used a thin back illuminated RCA CCD with $323 \times 512$ pixels of $30 \mu \mathrm{m}$ squared. In 1995, the detector was a Tektronix TK512 CCD with a pixel size of $27 \mu \mathrm{m}$. Aurélie was equipped with a Thomson TH7832 linear array with a pixel size of $13 \mu \mathrm{m}$ until 1999. From 2000 on, the detector of the Aurélie instrument was replaced by a $2048 \times 1024$ CCD EEV $42-20 \# 3$, with a pixel size of $13.5 \mu \mathrm{m}$ squared.

All the data were reduced in the standard way using the IHAP (for data taken before 1995) and MIDAS softwares developed at ESO.

We derived a template of the telluric absorption spectrum that affects the spectral ranges centered on $\mathrm{H} \alpha$ and $\mathrm{He}$ I $\lambda 5876$ from observations of a rapidely rotating star obtained at very different airmasses. This template was then used to achieve a first order correction of the telluric features on the spectra of HDE 228766. Finally, the spectra were normalized using properly chosen continuum windows.

Three spectra of HDE 228766 were obtained in October 2001 with the echelle spectrograph at the $2 \mathrm{~m}$ telescope of the Observatorio Astronómico Nacional of San Pedro Mártir (SPM). The detector was a Site CCD with $1024 \times 1024$ pixels of $24 \mu \mathrm{m}$ squared. The slit width was set to $150 \mu \mathrm{m}$ corresponding to $2^{\prime \prime}$ on the sky. The data were reduced using the MIDAS echelle package and the wavelength-calibrated spectra were rectified using the SPEFO code (Horn et al. 1996).

\section{The spectrum of HDE 228766}

As already pointed out by previous investigators, the spectrum of HDE 228766 displays a fair number of emission lines (see Fig. 1) that are essentially associated with the secondary (see Sect. 4). The most prominent ones are He II $\lambda \lambda 4686$, 10124; He I $\lambda$ 10830; H $\alpha$; $\mathrm{N}$ III $\lambda \lambda$ 4379, 4634, 4641; $\mathrm{N}$ Iv $\lambda \lambda 4058,7103-11,7123-29$ and Si IV $\lambda \lambda$ 4089, 4116. The spectral region from 4500 to $4540 \AA$ displays numerous faint $\mathrm{N}$ III emission lines, but we find no trace of the two "unidentified Of" emission lines at 4485 and $4504 \AA$ that were recently associated with S IV transitions (Werner \& Rauch 2001). The $\mathrm{H} \beta$ and $\mathrm{He}_{\mathrm{I}} \lambda 5876$ lines consist of a mixture of absorption and probably P-Cygni type emission lines. The presence of the $\mathrm{N} v \lambda \lambda$ 4603, 4620 lines is also a remarkable feature suggesting a very early spectral type (O3-O4) for the secondary (e.g. Walborn \& Fitzpatrick 1990; Walborn 2001). Beside
Table 1. Overview of our observing campaigns. The second column indicates the instrument used for the observations: $\mathrm{A}=$ Aurélie at the OHP $1.52 \mathrm{~m}, \mathrm{C}=$ Carelec at the OHP $1.93 \mathrm{~m}, \mathrm{SPM}=$ echelle spectrograph at the SPM $2 \mathrm{~m}$ telescope. $N$ yields the number of nights when HDE 228766 was observed.

\begin{tabular}{lccrc}
\hline \hline Campaign & $\begin{array}{c}\text { Inst. } \\
\end{array}$ & $\begin{array}{c}\text { Spectral range } \\
(\AA)\end{array}$ & $\begin{array}{r}\text { Recipr. disp. } \\
\left(\AA \mathrm{Am}^{-1}\right)\end{array}$ & $N$ \\
\hline Aug. 1989 & $\mathrm{C}$ & $6500-10400$ & 260 & 1 \\
Sep. 1989 & $\mathrm{C}$ & $8350-11150$ & 260 & 1 \\
Aug. 1990 & $\mathrm{C}$ & $6780-7200$ & 33 & 1 \\
Aug. 1990 & $\mathrm{C}$ & $8300-11100$ & 260 & 1 \\
Jul. 1995 & $\mathrm{C}$ & $4530-4720$ & 17 & 1 \\
Jul. 1999 & $\mathrm{A}$ & $4100-4950$ & 33 & 7 \\
Aug. 1999 & $\mathrm{A}$ & $4100-4950$ & 33 & 13 \\
Sep. 2000 & $\mathrm{A}$ & $4455-4905$ & 16 & 9 \\
Sep. 2000 & $\mathrm{A}$ & $6340-6790$ & 16 & 9 \\
Sep. 2000 & $\mathrm{A}$ & $5580-6030$ & 16 & 2 \\
Sep. 2000 & $\mathrm{A}$ & $3980-4440$ & 16 & 1 \\
Sep. 2001 & $\mathrm{A}$ & $6340-6790$ & 16 & 7 \\
Oct. 2001 & SPM & $3700-7370$ & 10 & 3 \\
\hline
\end{tabular}

the "typical" O-star absorption lines (e.g. H $\gamma$; He I $\lambda$ 4471; He II $\lambda \lambda 4200,4542 ; \ldots)$ there are a number of interstellar absorptions as well as diffuse interstellar bands (DIBs). The strongest interstellar lines in our data are $\mathrm{Na}$ I $\lambda \lambda$ 5890, 5896; $\mathrm{CH}^{+} \lambda 4233$ and $\mathrm{CH} \lambda 4300$.

The near-IR spectrum of HDE 228766 reveals a rather prominent and probably variable He II $\lambda 10124$ emission feature that was first reported by Vreux \& Andrillat (1979). Whereas this line is commonly observed in emission in the spectra of Wolf-Rayet (WR) stars, He II $\lambda 10124$ is usually seen in absorption in the spectra of Of stars. Emission in this line has been reported only for some of the hottest and most luminous Of stars. These include $\mathrm{Of}^{+}$stars (Vreux \& Andrillat 1979), several OIafpe transition objects (Crowther \& Bohannan 1996) as well as peculiar objects such as the Oef stars $\zeta$ Pup and $\lambda$ Cep and the high mass X-ray binaries Cyg X-1 (Conti \& Howarth 1999) and HD 153919 (Bohannan \& Crowther 1999). The strong He I $\lambda 10830$ emission was first noted by Andrillat \& Vreux (1979) and is also indicative of a large mass loss rate or an extreme wind extension (Conti \& Howarth 1999).

The equivalent width $(E W)$ ratios of the usual O-star classification lines He I $\lambda 4471$ and He II $\lambda 4542\left(W^{\prime}=\right.$ $E W(\mathrm{He}$ I $\lambda 4471) / E W(\mathrm{He}$ II $\lambda 4542)$, Conti 1973) point towards an $\mathrm{O} 7$ spectral type for both components $\left(\log W^{\prime}=\right.$ $-0.03 \pm 0.07$ and $\log W^{\prime}=-0.04 \pm 0.16$ for the primary and secondary respectively). The presence of $\mathrm{Si}$ iv $\lambda \lambda 4089,4116$ in emission in addition to the "classical" $\mathrm{N}$ III $\lambda \lambda$ 4634-41 and $\mathrm{He}$ II $\lambda 4686$ Of emission lines indicates an $\mathrm{Of}^{+}$classification (see e.g. Walborn \& Fitzpatrick 1990) for the secondary star. Though these results are in crude agreement with the classification proposed by MC77 (O7.5 + O5.5f) based on photographic spectra, we caution that the spectral types are rather uncertain due to the serious blending of the classification lines of both stars. Moreover, an $\mathrm{O} 7$ classification for the secondary 


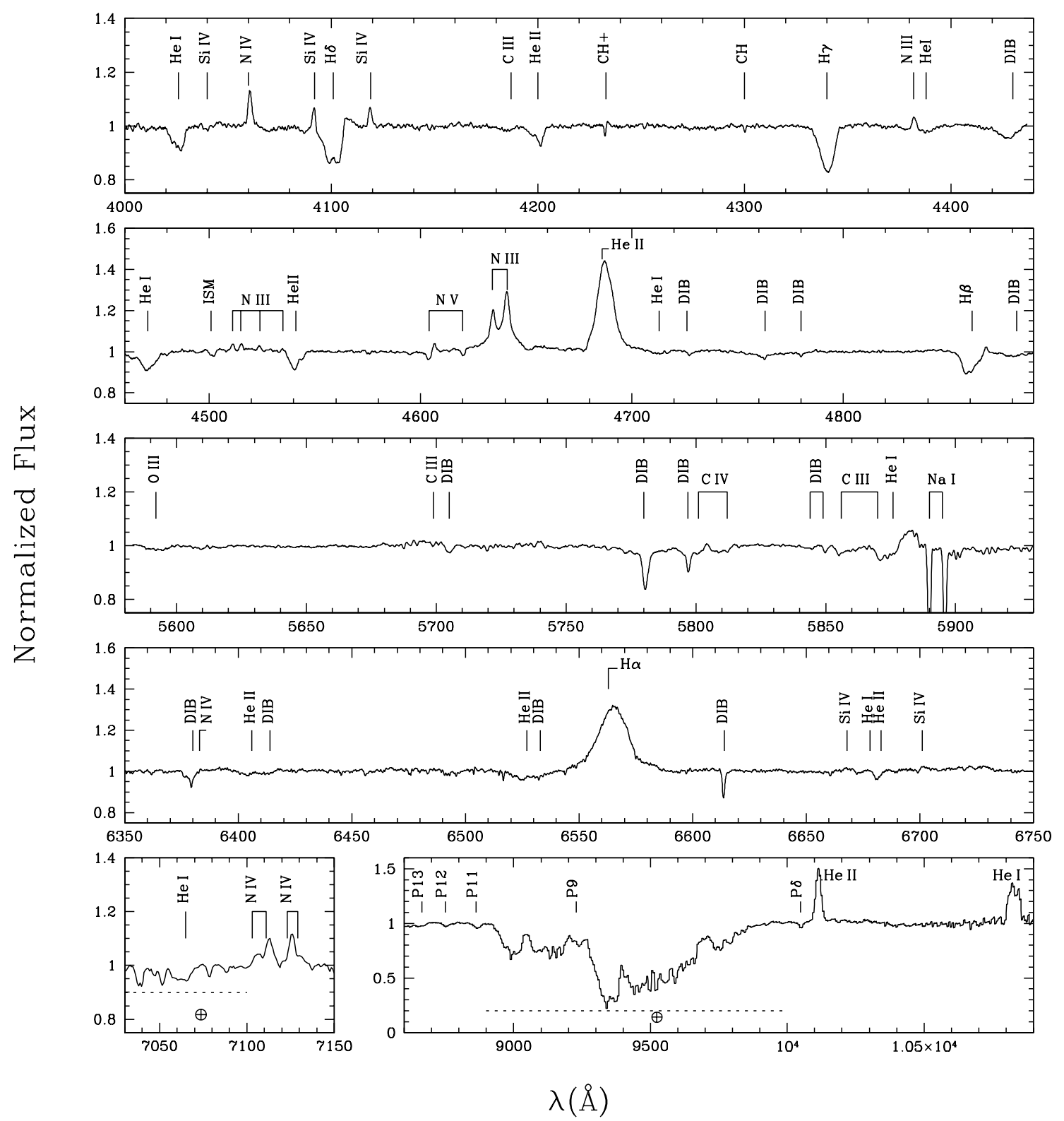

Fig. 1. Composite spectrum of HDE 228766 from the near ultraviolet to the near infrared. All the observations shown here were taken in September 2000 except for the spectral ranges 7030-7150 and 8600-10 900 that were observed in August 1990. The most prominent stellar and interstellar features are identified. The $\oplus$ symbol indicates spectral regions that are considerably affected by telluric absorptions and for which no first order correction was applied.

is obviously at odds with the early spectral type implied by the presence of the $\mathrm{N}$ IV and $\mathrm{N} v$ lines (see also Sect. 6.1).

At this stage, it is worth pointing out that the He I $\lambda 4471$ absorption is likely to be affected by wind emission. In fact, the $\mathrm{He}$ I $\lambda 5876$ line appears as a mixture of emission and absorption features and a slightly weaker emission at $\mathrm{He}$ I $\lambda 4471$ could essentially remain undetected though it would affect the $E W \mathrm{~s}$ of this line in the spectrum of both stars. The He II $\lambda 4542$ line in the spectrum of the secondary might not be of purely photospheric origin either. Indeed, our orbital solution (see the forthcoming Sect. 4) reveals a surprisingly large difference of $150 \mathrm{~km} \mathrm{~s}^{-1}$ between the apparent systemic velocities of the two stars with the secondary being the star with the more negative $\gamma$. Since most of the emission lines are also associated with the secondary, the blueshift of the secondary's He II $\lambda 4542$ absorption could be the result of the strong wind of this star. In this context, Crowther \& Bohannan (1997) showed that in extreme Of stars, the usual diagnostic lines such as He II $\lambda \lambda 4542$, 5412 are significantly affected by wind contamination and as a consequence the distinction between the most extreme Of stars and the least extreme WN stars becomes a rather tricky issue. It is worth noting that Hackwell et al. (1974) did not find a significant infrared excess in the spectrum of HDE 228766. Such an excess is often seen for Wolf-Rayet stars and is attributed to free-free emission from the dense stellar wind. The relative weakness of the infrared free-free emission in HDE 228766 is consistent with an Of or a weak-lined WN spectral type for the secondary. 


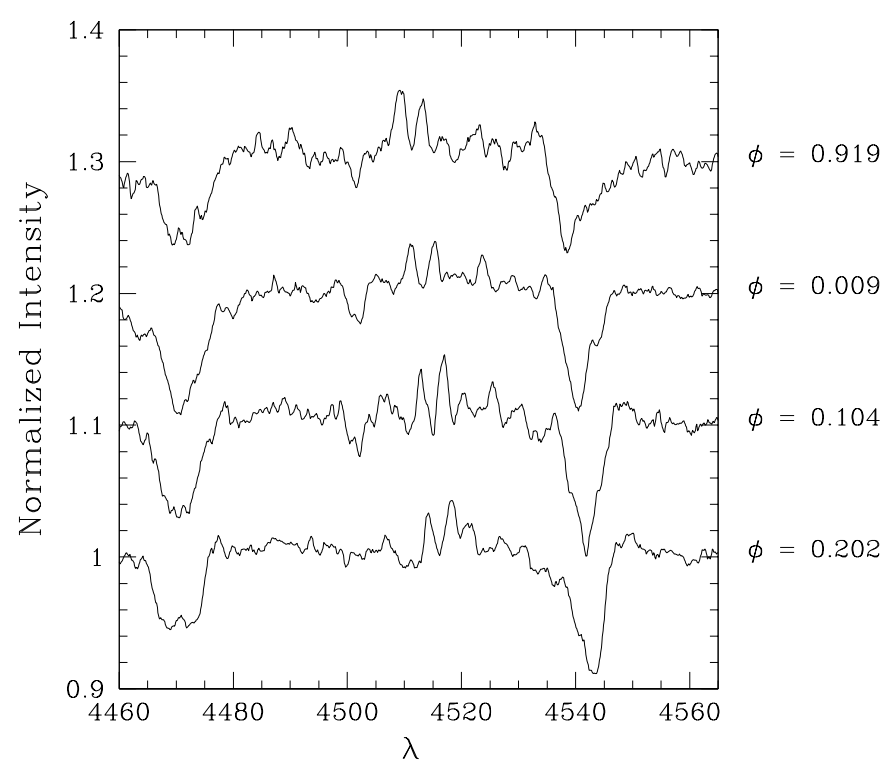

Fig. 2. Examples of the blue-violet spectra of HDE 228766 as a function of phase illustrating the severe blending of the absorption lines even around $\phi=0.2$. Note also the numerous weaker $\mathrm{N}$ III emission lines.

\section{Orbital solution}

The most extensive and most accurate compilation of radial velocities $(R V \mathrm{~s})$ of HDE 228766 in the literature concerns the He II $\lambda 4686$ emission line. We measured the $R V$ s of this line on our spectra by fitting a Gaussian. Adopting the orbital period of $10.7424 \mathrm{~d}$ as derived by MC77, we used our He II $\lambda 4686$ $R V$ s to determine a new orbital solution for this line (see Rauw et al. 2002). Apart from a small phase-shift, this new solution is in excellent agreement with the results of Hart (1957) and MC77. This finding prompted us to take advantage of the entire $R V$ dataset of $\mathrm{He}$ II $\lambda 4686$ available in the literature to derive a better estimate of the orbital period of HDE 228766. We applied the generalized spectrogram technique of Heck et al. (1985; see also Gosset et al. 2001) and the trial method of Lafler \& Kinman (1965) to this timeseries and both methods consistently yield $10.7426 \mathrm{~d}$ as the best estimate for $P_{\text {orb }}$. Assuming that the uncertainty on this result amounts to one tenth of the natural width of the peak in the periodogram, we estimate an uncertainty of $6 \times 10^{-4} \mathrm{~d}$. Therefore, our new orbital period confirms the previous determinations. In the following, all the orbital solutions are computed adopting our new value of $10.7426 \mathrm{~d}$.

A close inspection of our data reveals that most of the absorption lines of the binary components remain blended over the main part of the orbital cycle (Fig. 2). The only absorption feature that seems reasonably well suited to establish an orbital solution for both stars is the He II $\lambda 4542$ absorption line. The He I $\lambda 4471$ and $\mathrm{H} \gamma$ absorptions are seldom completely deblended and suffer most probably from a contamination by a variable wind emission.

To determine the radial velocities of the He II $\lambda 4542 \mathrm{ab}-$ sorption lines, we have built a template of the line in the spectrum of each star by fitting two Gaussians to the blend around

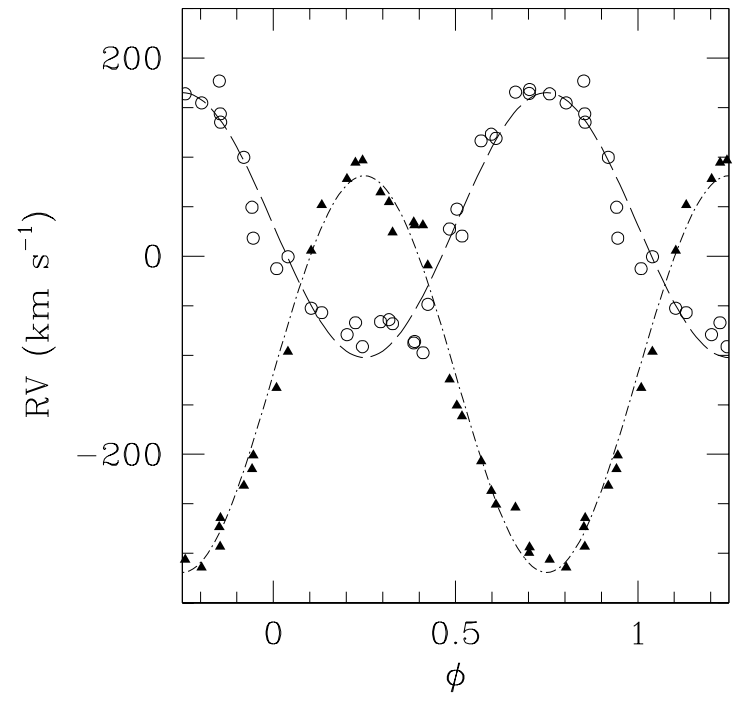

Fig. 3. Radial velocity curve of the HDE 228766 binary system as derived from the He II $\lambda 4542$ absorption line (see Table 3). Open circles and filled triangles stand for the primary and secondary $R V \mathrm{~s}$ respectively.

maximum separation. At the other orbital phases, we used these templates within a cross-correlation like technique to deblend the lines of the two components. We define phase 0.0 as the phase of conjunction with the secondary star being in front of the primary and we adopt the $10.7426 \mathrm{~d}$ orbital period derived above.

Allowing for an eccentric orbital solution yields $e=0.06 \pm$ 0.02 for the $R V \mathrm{~s}$ derived from the He II $\lambda 4542$ absorption lines. However, the radial velocity curves of the He II $\lambda 4686$ and $\mathrm{N}$ III $\lambda \lambda$ 4634, 4641 emission lines all indicate an eccentricity below 0.03 with correspondingly large errors $(\sim 0.01,1 \sigma)$. Therefore, although we cannot completely rule out a very small eccentricity, in the following we assume $e=0.0$.

Our He II $\lambda 4542$ radial velocity measurements yield a significantly larger mass ratio $(q=1.50)$ than the value $(q=$ 1.04) obtained by MC77. This larger mass ratio results from a slightly lower $K_{1}\left(134 \mathrm{vs} .150 \mathrm{~km} \mathrm{~s}^{-1}\right)$ and a significantly larger $K_{2}$ (200 vs. $\left.156 \mathrm{~km} \mathrm{~s}^{-1}\right)$. We find a huge difference of $150 \mathrm{~km} \mathrm{~s}^{-1}$ between the apparent systemic velocities of the two components of the system with the secondary line being blue-shifted with respect to the primary. Our non-LTE analysis of the spectrum of HDE 228766 (Sect. 6.1 below) confirms that this shift of the apparent $\gamma$-velocities can be ascribed to wind contamination of the secondary's helium lines. This blueshift of the secondary's absorption was already discovered by Massey \& Conti (1977) but these authors reported a difference of "only" $45 \mathrm{~km} \mathrm{~s}^{-1}$. MC77 used the mean $R V$ s of several absorption lines to derive their orbital solution. Due to the severe blending problems, these authors cautioned that their $R V$ measurements of the absorption lines on their photographic plates were at least partly subjective. Our $R V \mathrm{~s}$ obtained through a cross-correlation technique are in principle less subjective, but refer to the sole He II $\lambda 4542$ line thus rendering a direct comparison of our $\gamma$ velocities with the ones of MC77 difficult. 
Table 2. Journal of observations of HDE 228766 in the blue-violet range obtained with the Carelec and Aurélie spectrograph at OHP. The orbital phases are computed with respect to HJD 2451822.052 with a period of $10.7426 \mathrm{~d}$ (see Table 3). The last column yields the equivalent width of the He II $\lambda 4686$ emission line integrated from 4665 to $4705 \AA$.

\begin{tabular}{|c|c|c|c|c|c|c|c|}
\hline \multirow[t]{2}{*}{ HJD-2 440000} & \multirow[t]{2}{*}{$\phi$} & \multicolumn{2}{|c|}{ Не пг $\lambda 4542$} & \multirow{2}{*}{$\begin{array}{l}\mathrm{N} \text { III } \lambda 4634 \\
R V\left(\mathrm{~km} \mathrm{~s}^{-1}\right)\end{array}$} & \multirow{2}{*}{$\begin{array}{l}\mathrm{N}_{\text {III }} \lambda 4641 \\
R V\left(\mathrm{~km} \mathrm{~s}^{-1}\right)\end{array}$} & \multicolumn{2}{|c|}{ Не II $\lambda 4686$} \\
\hline & & $R V_{1}\left(\mathrm{~km} \mathrm{~s}^{-1}\right)$ & $R V_{2}\left(\mathrm{~km} \mathrm{~s}^{-1}\right)$ & & & $R V\left(\mathrm{~km} \mathrm{~s}^{-1}\right)$ & $E W(\AA)$ \\
\hline 9917.412 & .702 & 164.2 & -299.4 & -224.6 & -222.0 & -190.2 & -5.33 \\
\hline 11373.492 & .245 & -91.3 & 96.7 & 221.3 & 196.6 & 283.8 & -5.61 \\
\hline 11374.379 & .327 & -68.2 & 24.0 & 178.6 & 168.6 & 254.7 & -5.72 \\
\hline 11375.415 & .424 & -48.7 & -9.3 & 96.1 & 67.3 & 159.7 & -5.49 \\
\hline 11376.425 & .518 & 20.3 & -161.4 & -19.9 & -47.1 & 32.5 & -5.22 \\
\hline 11377.428 & .611 & 119.0 & -250.7 & -153.0 & -171.2 & -107.5 & -5.60 \\
\hline 11378.417 & .703 & 168.3 & -293.9 & -224.0 & -244.5 & -187.2 & -5.74 \\
\hline 11379.494 & .803 & 154.7 & -314.1 & -220.2 & -236.7 & -172.6 & -5.57 \\
\hline 11396.488 & .385 & -87.6 & 34.3 & 142.3 & 125.5 & 214.3 & -5.49 \\
\hline 11397.543 & .484 & 27.5 & -124.5 & 9.9 & -14.9 & 80.6 & -4.99 \\
\hline 11398.473 & .570 & 116.4 & -207.1 & -107.2 & -120.4 & -50.2 & -5.66 \\
\hline 11399.486 & .664 & 165.6 & -253.7 & -207.7 & -218.1 & -159.3 & -5.36 \\
\hline 11401.537 & .855 & 135.2 & -264.2 & -185.8 & -207.2 & -141.1 & -5.53 \\
\hline 11402.465 & .942 & 49.3 & -214.9 & -98.6 & -118.9 & -36.5 & -5.34 \\
\hline 11403.524 & .040 & -0.6 & -96.5 & 39.3 & 15.2 & 119.0 & -5.36 \\
\hline 11404.518 & .133 & -57.0 & 51.7 & 151.4 & 124.8 & 236.3 & -5.43 \\
\hline 11405.514 & .226 & -67.2 & 94.4 & 207.7 & 189.3 & 298.0 & -5.52 \\
\hline 11406.500 & .317 & -64.2 & 54.5 & 208.5 & 190.8 & 266.5 & -5.61 \\
\hline 11407.503 & .411 & -97.5 & 31.1 & 106.0 & 88.3 & 197.9 & -5.24 \\
\hline 11408.503 & .504 & 47.4 & -150.7 & -6.5 & -30.5 & 54.6 & -5.21 \\
\hline 11409.517 & .598 & 123.0 & -236.8 & -138.5 & -163.3 & -101.8 & -5.88 \\
\hline 11810.440 & .919 & 99.7 & -231.7 & -122.5 & -123.3 & -71.4 & -5.88 \\
\hline 11811.408 & .009 & -12.6 & -132.8 & 2.5 & -3.4 & 96.1 & -4.36 \\
\hline 11812.427 & .104 & -52.5 & 5.6 & 122.8 & 120.8 & 191.0 & -5.81 \\
\hline 11813.484 & .202 & -79.2 & 77.9 & 200.9 & 202.5 & 281.1 & -5.78 \\
\hline 11814.469 & .294 & -66.2 & 64.5 & 210.3 & 210.0 & 279.2 & -5.92 \\
\hline 11815.473 & .388 & -86.2 & 31.3 & 142.1 & 137.8 & 216.9 & -5.77 \\
\hline 11819.453 & .758 & 163.8 & -306.2 & -234.4 & -233.3 & -202.2 & -5.99 \\
\hline 11820.460 & .852 & 176.8 & -273.4 & -194.9 & -180.0 & -143.2 & -5.71 \\
\hline 11820.487 & .854 & 143.7 & -293.3 & -186.9 & -181.3 & -144.4 & -5.43 \\
\hline 11821.461 & .945 & 18.2 & -201.0 & -89.4 & -87.7 & -20.5 & -5.66 \\
\hline
\end{tabular}

We have measured the radial velocities of the $\mathrm{N}$ III $\lambda \lambda 4634$, 4641 and He II $\lambda 4686$ emission lines (Table 2). The lines appear to follow closely the orbital motion of the secondary star without any significant phase lag (Table 3). The semiamplitude of our $\mathrm{N}$ III $R V$-curves overlap within $3 \mathrm{~km} \mathrm{~s}^{-1}$ with previous results, whereas the semi-amplitude of our He II $\lambda 4686 R V$-solution (244 $\mathrm{km} \mathrm{s}^{-1}$ ) is intermediate between the values of 218 and $260 \mathrm{~km} \mathrm{~s}^{-1}$ obtained respectively by MC77 and Hart (1957). It is worth mentioning that the absorption components of the $\mathrm{N} v \lambda \lambda 4604,4620$ lines as well as the $\mathrm{N} v \lambda 4604 \mathrm{P}$-Cygni emission also follow the motion of the secondary star with a semi-amplitude of 223-227 $\mathrm{km} \mathrm{s}^{-1}$ (see also Table 4 below).

A tricky question that occurs frequently for binary systems harboring Wolf-Rayet or extreme Of stars is which of the spectral lines reflects the actual orbital motion of the WR or Of star. In the case of HDE 228766, it seems likely that the absorption lines of the secondary are formed in the stellar wind rather than in the photosphere. The $\gamma$ velocity of the He II $\lambda 4542$ absorption is blue-shifted by more than $100 \mathrm{~km} \mathrm{~s}^{-1}$ with respect to the $R V$-curve of the $\mathrm{N}$ III emission lines. This blue-shift suggests that the absorption forms in the wind, where the outflow has already reached larger velocities than in the formation area of the narrow emission lines. This situation is reminiscent of the case of the WN7ha + O binary WR 22 for which the H9 absorption appears blue-shifted by $80 \mathrm{~km} \mathrm{~s}^{-1}$ with respect to the $\mathrm{N}$ IV $\lambda 4058$ emission (Rauw et al. 1996). For WR 22, it was found that the narrow $\mathrm{N}$ IV emission provides the best indicator of the orbital motion of the WN7ha star. It seems likely that the same holds for the $\mathrm{N}$ III, $\mathrm{N}$ IV and $\mathrm{N} v$ emission lines of the secondary in HDE 228766. Indeed, the various narrow emission lines yield the same $K$ and their $\gamma$ velocities are in reasonable agreement with that of the primary's He II $\lambda 4542$ absorption line.

The line formation region of the He II $\lambda 4686$ emission is probably more extended and hence more affected by the presence of the primary and/or by a wind interaction phenomenon than that of the nitrogen lines. 
Table 3. New orbital solutions for HDE 228766. The $R V$ s of the emission lines vary almost perfectly in phase with the $R V$ of the secondary's He II $\lambda 4542$ absorption line. $T_{0}$ refers to the time of conjunction with the primary being behind. $R_{\mathrm{RL}}$ stands for the radius of a sphere with a volume equal to that of the Roche lobe computed according to the formula of Eggleton (1983).

\begin{tabular}{|c|c|c|c|c|c|}
\hline & \multicolumn{2}{|c|}{ He II $\lambda 4542$} & \multirow[t]{2}{*}{$\mathrm{N}_{\text {III }} \lambda 4634$} & \multirow[t]{2}{*}{$\mathrm{N}_{\text {III }} \lambda 4641$} & \multirow[t]{2}{*}{ Не ІІ $\lambda 4686$} \\
\hline & Primary & Secondary & & & \\
\hline$P$ (days) & \multicolumn{2}{|c|}{10.7426 (fixed) } & \multicolumn{3}{|c|}{ (fixed) } \\
\hline$e$ & \multicolumn{2}{|c|}{0.0 (adopted) } & & 0.0 (adopted) & \\
\hline$T_{0}(\mathrm{HJD}-2450000)$ & \multicolumn{2}{|c|}{$1822.052 \pm 0.305$} & $1822.081 \pm 0.044$ & $1822.034 \pm 0.100$ & $1822.040 \pm 0.072$ \\
\hline$\gamma\left(\mathrm{km} \mathrm{s}^{-1}\right)$ & $31.4 \pm 7.3$ & $-119.0 \pm 8.2$ & $-10.1 \pm 0.7$ & $-22.4 \pm 1.5$ & $52.0 \pm 1.2$ \\
\hline$K\left(\mathrm{~km} \mathrm{~s}^{-1}\right)$ & $133.7 \pm 7.6$ & $200.2 \pm 10.9$ & $224.9 \pm 1.0$ & $222.1 \pm 2.1$ & $244.5 \pm 1.7$ \\
\hline$a \sin i\left(R_{\odot}\right)$ & $28.4 \pm 1.6$ & $42.5 \pm 2.3$ & $47.7 \pm 0.2$ & $47.1 \pm 0.4$ & $51.9 \pm 0.4$ \\
\hline$q=m_{1} / m_{2}$ & \multicolumn{2}{|c|}{$1.50 \pm 0.11$} & & & \\
\hline$m \sin ^{3} i\left(M_{\odot}\right)$ & $24.8 \pm 1.8$ & $16.6 \pm 2.4$ & & & \\
\hline$R_{\mathrm{RL}} \sin i\left(R_{\odot}\right)$ & $29.3 \pm 1.5$ & $24.4 \pm 1.2$ & & & \\
\hline
\end{tabular}

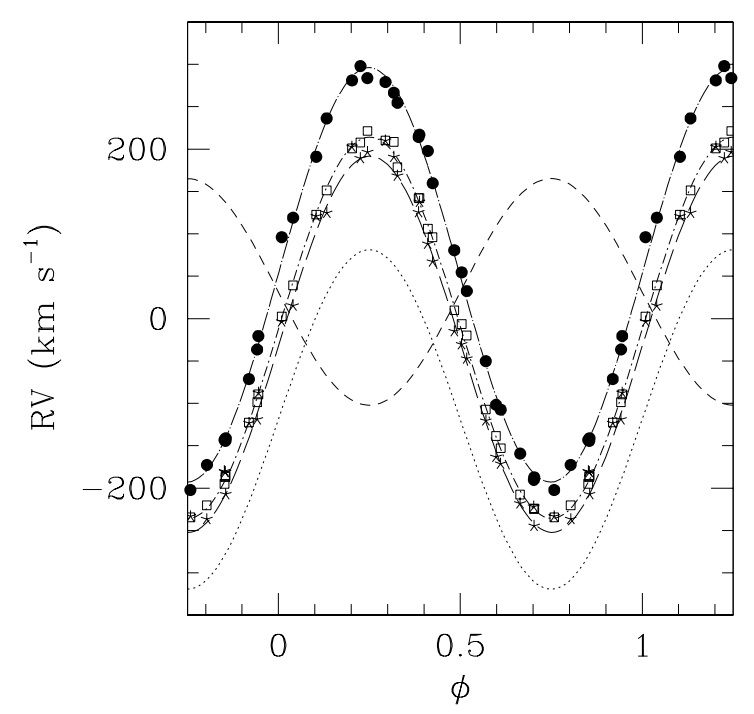

Fig. 4. Radial velocity curves of the $\mathrm{N}$ III $\lambda 4634$ (open squares), $\mathrm{N}$ III $\lambda 4641$ (stars) and He II $\lambda 4686$ (black dots) emission lines in the spectrum of HDE 228766. The He in $\lambda 4542$ orbital solutions of the primary (dashed line) and secondary (dotted line) are shown for comparison.

Therefore, we suggest that the true $K_{2} \simeq K_{\mathrm{N} \text { III }} \simeq$ $225 \mathrm{~km} \mathrm{~s}^{-1}$. Adopting this value yields a mass ratio of $q=$ 1.67, a projected separation of $\left(a_{1}+a_{2}\right) \sin i=76 R_{\odot}$ and minimum masses of $m_{1} \sin ^{3} i=31.7$ and $m_{2} \sin ^{3} i=$ $25.5 M_{\odot}$ for the primary and secondary respectively. The rather large minimum masses suggest that the inclination $i$ could be large and HDE 228766 might display photometric variability. Unfortunately, it seems that there has been no photometric investigation of this system so far and no light-curve is available that could allow to constrain $i$.

\section{Line profile variability}

\subsection{He $\| \lambda 4686$}

The He II $\lambda 4686$ line appears as a strong emission with a nearly symmetric profile, the blue wing being only slightly steeper than the red wing. The full width at half maximum of the line amounts to about $7 \AA$ (i.e. $\sim 450 \mathrm{~km} \mathrm{~s}^{-1}$ ).
This line displays no significant phase-locked line profile variations contrary to what is observed in several other interacting wind Of binary systems (e.g. Rauw et al. 1999; Sana et al. 2001). There are however some intensity variations. We have measured the equivalent width of the line integrated between 4665 and $4705 \AA$. There is a large scatter in the data points, without any clear dependence on the orbital phase (though the lowest $E W \mathrm{~s}$ correspond to the two conjunction phases). The mean $E W$ of the He II $\lambda 4686$ emission is $-5.52 \AA$ with variations between -4.36 and $-5.99 \AA$ (see Table 2). Despite this large peak to peak variation, the $1 \sigma$ dispersion on the mean $E W$ is only $0.32 \AA$, i.e. $6 \%$. This result is in good agreement with the assertion of Massey \& Conti (1977) that the $E W$ of this line does not show variations of more than $15 \%$.

Though the emission peak follows the orbital motion of the secondary, there could be higher order variations in the line profile reflecting an interaction with the primary. Unveiling such variations requires an analysis of the entire profile. For this purpose, we have applied a Doppler tomography technique to map the line formation region of the He II $\lambda 4686$ emission line in velocity space (see e.g. Thaller et al. 2001 for an example of the application of this method to early-type binaries). Our method uses a Fourier filtered back projection algorithm (Horne 1991). The radial velocity of any gas flow that is stationary in the rotating frame of reference of the binary can be described by a so-called "S-wave" relation:

$v(\phi)=-v_{x} \cos (2 \pi \phi)+v_{y} \sin (2 \pi \phi)+v_{z}$

$\phi$ stands for the orbital phase, whereas $\left(v_{x}, v_{y}, v_{z}\right)$ are the velocity coordinates of the gas flow. The S-wave relation assumes that the $x$-axis runs between the stars, from the primary to the secondary, whilst the positive $y$-axis points in the direction of the secondary's orbital motion. Finally, the $z$-axis is perpendicular to the orbital plane. The Doppler map consists of a projection of the S-wave relation on the $\left(v_{x}, v_{y}\right)$ plane. Therefore, each pixel in a Doppler map, specified by its velocity coordinates is associated with a particular S-wave (see e.g. Horne 1991 for a detailed discussion of the method).

Our data were weighted so as to account for the uneven sampling of the orbital cycle. The resulting Doppler map (Fig. 5) reveals an extended line formation region centered on the secondary without any obvious trace of a structure that 


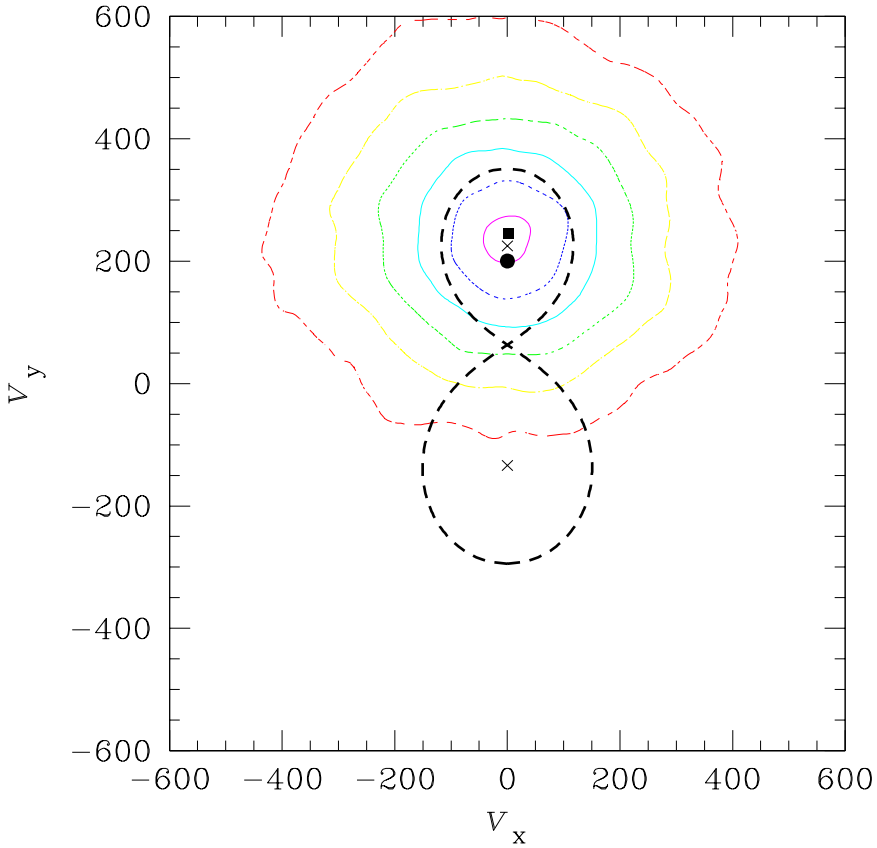

Fig. 5. Doppler map of the He II $\lambda 4686$ emission line in the spectrum of HDE 228766. The crosses correspond to the $R V \mathrm{~s}$ of the center of mass of the primary and secondary as derived from the $\mathrm{He}$ iा $\lambda 4542$ absorption and $\mathrm{N}$ III $\lambda 4634$ emission respectively. The shape of the Roche lobe in velocity-space (thick dashed line) has been calculated for a mass ratio of 1.67. The black dot and the black square correspond to the $R V$ curves of the secondary's He II $\lambda 4542$ absorption and the peak of the He II $\lambda 4686$ emission line respectively. The Doppler map was computed with $v_{z}$ set to $52 \mathrm{~km} \mathrm{~s}^{-1}$.

could be attributed to a wind interaction or a Roche lobe overflow. Therefore, it seems likely that the bulk of the He II $\lambda 4686$ emission arises in a relatively unperturbed part of the stellar wind of the secondary.

\section{2. $H \beta$}

The profile variations of the $\mathrm{H} \beta$ line are clearly phase-locked and stable over time scales of at least two years. The line displays a weak P-Cygni emission component between phases $\phi=0.0$ and $\phi=0.5$, i.e. when the lines of the secondary are red-shifted (see Fig. 6). The emission component vanishes when the secondary's lines are blue-shifted. This situation strongly suggests that the emission component is associated with the secondary star and that the bulk of the profile variations result from blending effects with the primary's absorption line.

\section{3. $H \alpha$}

Figure 7 reveals $\mathrm{H} \alpha$ emission consisting of at least two components. The stronger component roughly follows the orbital motion of the secondary whilst the weaker component moves roughly in anti-phase. The two emission components are best seen at quadrature phases.

MC77 reported the presence of a blue-shifted absorption feature in the $\mathrm{H} \alpha$ line near $\phi \sim 0.3$. Although we do not observe

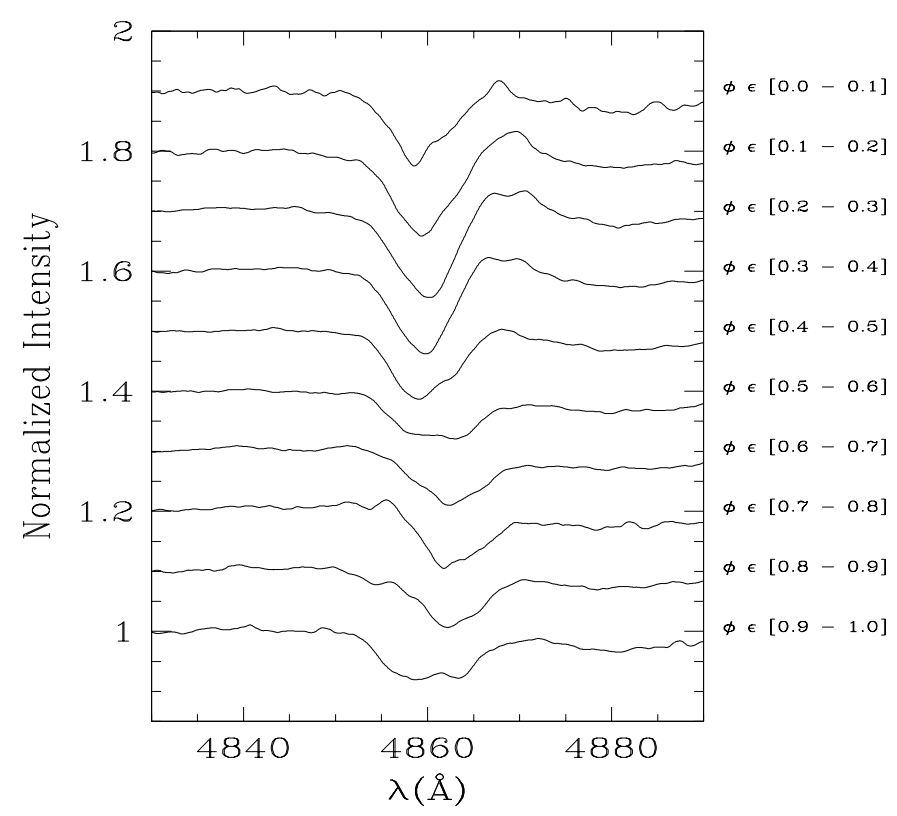

Fig. 6. Normalized profile of the $\mathrm{H} \beta$ line in the spectrum of HDE 228766 as observed in July and August 1999. The various spectra have been binned into phase intervals of 0.1 . The broad absorption around 4880 is due to a diffuse interstellar band.

an absorption feature that goes below the continuum level, we note that the double-peaked structure of the line around $\phi \sim 0.3$ could be interpreted as a blend of a blue-shifted absorption (associated with the primary) with a red-shifted emission (belonging to the secondary). However, in this case, we would expect the $\mathrm{H} \alpha$ profile to display a double-peaked profile with a roughly central absorption at conjunction phases, which is not observed. Therefore, it seems more likely that the weaker component seen near quadrature corresponds to a genuine emission component that moves independently of the stronger component.

The Doppler map of this line (Fig. 8) reveals a more complex structure than in the case of He II $\lambda$ 4686. The bulk of the emission still follows the secondary star though with a significantly larger $R V$ amplitude. There is a secondary "peak" in the map associated with the weaker emission component seen in the spectra. This structure does not seem to belong to any of the stars. The equivalent width of this feature usually corresponds to less than $15 \%$ of the $E W$ of the total $\mathrm{H} \alpha$ emission. The remaining $\sim 85 \%$ of the flux follow the motion of the secondary star.

Finally, we have also carried out a simple $\mathrm{S}$-wave analysis of the $R V \mathrm{~s}$ of the peaks of the various emission lines in the spectrum of HDE 228766, i.e. we fitted the $R V \mathrm{~s}$ of the peaks following Eq. (1). The results are given in Table 4. We note that the weaker $\mathrm{H} \alpha$ component is the only spectral feature that presents a significant $v_{x}$ component and has a negative $v_{y}$. All other lines investigated in Table 4 display an orbital motion consistent with line formation in the wind of the secondary. 


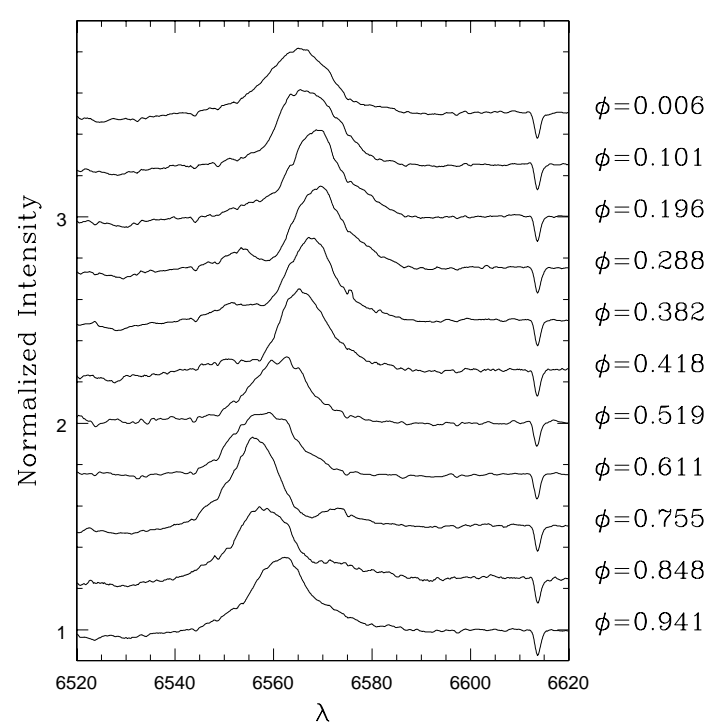

Fig. 7. Montage of the $\mathrm{H} \alpha$ line profile variability. All data are from the September 2000 campaign apart from the spectra at $\phi=0.418$ (Sep. 2001) and $\phi=0.519,0.611$ (Oct. 2001).

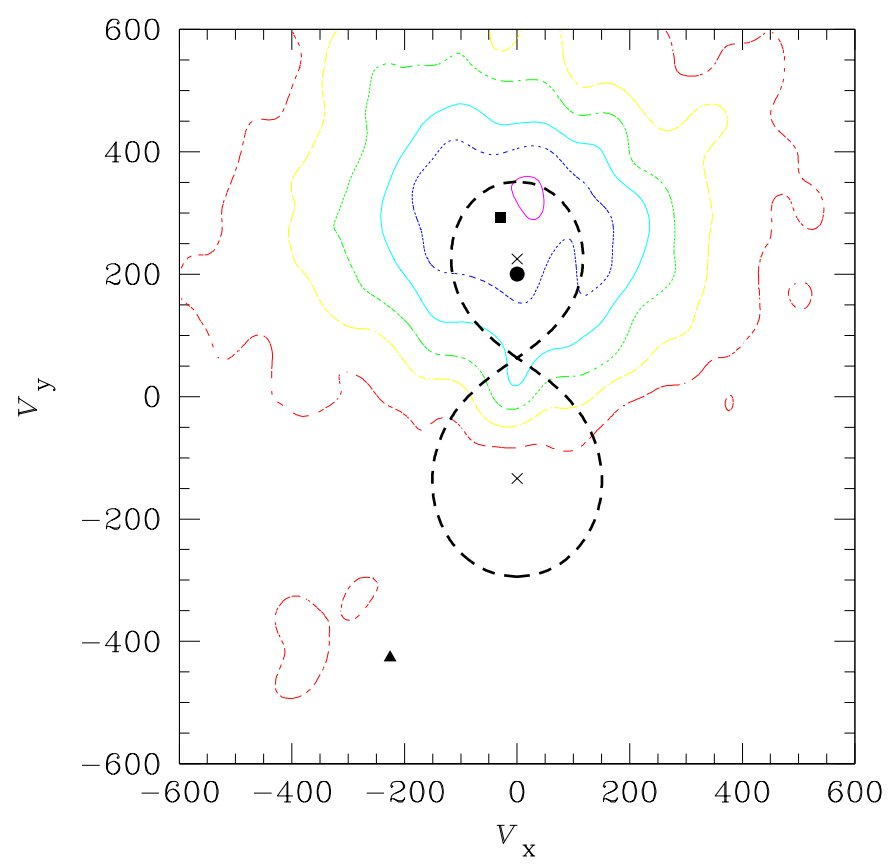

Fig. 8. Same as Fig. 5, but for the $\mathrm{H} \alpha$ emission with $v_{z}$ set to $-8.3 \mathrm{~km} \mathrm{~s}^{-1}$. The symbols have the same meaning as before except for the filled square and the filled triangle that stand for the S-waves of the dominant peak (filled square) and the secondary peak (filled triangle) of the $\mathrm{H} \alpha$ profile.

\section{Discussion}

\subsection{The fundamental parameters of HDE 228766}

Massey \& Conti (1977) inferred the relative brightness of the components of HDE 228766 from the ratio of the intensities of their $\mathrm{H} 8$ and $\mathrm{H} 9$ lines. In this way, they derived a roughly equal brightness $\left(\Delta m_{V} \leq 0.5 \mathrm{mag}\right)$. We used the $E W \mathrm{~s}$ of the He I $\lambda 4471$ and He II $\lambda 4542$ lines as measured on the spectra of maximum $R V$-separation and compared them with the $E W \mathrm{~s}$
Table 4. Summary of the S-wave analysis of the $R V \mathrm{~s}$ of the peaks of various emission features in the spectrum of HDE 228766. The absorption components of the $\mathrm{N} v \mathrm{P}$-Cygni profiles are also included. The $\left(v_{x}, v_{y}, v_{z}\right)$ velocity components and the mean $|\mathrm{O}-\mathrm{C}|$ residuals are given in $\mathrm{km} \mathrm{s}^{-1}$.

\begin{tabular}{lrrrr}
\hline \hline Line & \multicolumn{1}{c}{$v_{x}$} & \multicolumn{1}{c}{$v_{y}$} & \multicolumn{1}{c}{$v_{z}$} & $\overline{|\mathrm{O}-\mathrm{C}|}$ \\
\hline $\mathrm{H} \alpha$ (strong) & -29.1 & 292.2 & -8.3 & 23.2 \\
$\mathrm{H} \alpha$ (weak) & -225.3 & -426.3 & -51.2 & 60.3 \\
$\mathrm{He}$ II $\lambda 4686$ & -1.7 & 244.5 & 52.0 & 9.1 \\
$\mathrm{~N}$ III $\lambda 4511$ & 16.1 & 224.6 & 0.4 & 8.3 \\
$\mathrm{~N}$ III $\lambda 4515$ & 10.3 & 220.1 & 0.0 & 10.9 \\
$\mathrm{~N}$ III $\lambda 4634$ & 3.8 & 224.9 & -10.1 & 5.1 \\
$\mathrm{~N}$ III $\lambda 4641$ & -2.3 & 222.1 & -22.4 & 11.5 \\
$\mathrm{~N}$ v $\lambda 4604$ (A) & 4.0 & 226.5 & -37.6 & 9.1 \\
$\mathrm{~N}$ v $\lambda 4604$ (E) & 5.8 & 222.8 & 165.8 & 9.9 \\
$\mathrm{~N}$ v $\lambda 4620$ (A) & 3.2 & 223.3 & -12.6 & 11.4 \\
\hline
\end{tabular}

of single O-stars quoted by Conti \& Alschuler (1971). The results depend of course on the (rather uncertain) spectral types adopted for each star, but they essentially confirm the results of MC77: the line ratios suggest in fact that both stars have more or less the same brightness with the secondary being somewhat brighter $\left(m_{V, \mathrm{p}}-m_{V, \mathrm{~s}} \sim 0.3\right)$.

Unfortunately, the distance of HDE 228766 is extremely uncertain. Popova \& Kraicheva (1984) associated the system with the open cluster Dolidze 3. Zakirov (1999) determined a distance of $1.9 \mathrm{kpc}$ for the eclipsing binary KV Cyg near the center of Dolidze 3. However, the membership of HDE 228766 in Dolidze 3 seems questionable since the system lies at $\sim 38^{\prime}$ from the cluster center (according to Ruprecht et al. 1983). On the other hand, HDE 228766 lies in the region of the sky spanned by the Cyg OB1 association for which Humphreys (1978) quoted a mean distance of $1.82 \mathrm{kpc}$. Let us start by assuming that HDE 228766 lies indeed at a distance of $1.9 \mathrm{kpc}$. Adopting the $V=9.14$ magnitude and $B-V=0.65$ colour $\left(E_{B-V} \simeq 0.95\right)$ quoted by Hiltner (1956) and assuming a ratio of total-to-selective extinction $R=3.2$, we find that the absolute magnitude of HDE 228766 should be -5.29 . Note that the magnitude (9.15) and colour (0.60) found by Demers \& Fernie (1964) yield an absolute magnitude of -5.12 . These absolute magnitudes are at odds with the idea that HDE 228766 consists of two O-type giants or supergiants which should rather have a combined absolute magnitude of $\sim-6.6$ to $\sim-7.2$ (SchmidtKaler 1982). It appears rather unlikely that a peculiar extinction law could account for the entire discrepancy. Therefore, it seems more plausible that the actual distance of the system is larger than $1.9 \mathrm{kpc}$. For instance, if we assume an absolute magnitude of $M_{V, \mathrm{~s}}=-6.0$ for the secondary and $M_{V, \mathrm{p}}=-5.7$ for the primary, we obtain a distance of $3.5 \mathrm{kpc}$ for HDE 228766.

To determine the stellar properties of HDE 228766 we have modelled the combined spectrum of HDE 228766 by means of model atmosphere codes. For the primary's photospheric spectrum we have adopted a TLUSTY non-LTE model (Hubeny \& Lanz 1995) corresponding to the O8 III star $\lambda$ Ori. The relevant 
model parameters are $T_{\text {eff, }}=36000 \mathrm{~K}, R_{*, \mathrm{p}}=16 R_{\odot}\left(L_{\mathrm{bol}, \mathrm{p}}=\right.$ $\left.380000 L_{\odot}\right), \log g=3.5$ and $v \sin i=200 \mathrm{~km} \mathrm{~s}^{-1}$. At this stage, it is worth emphasizing that the value of the projected rotational velocity of the primary has to be significantly larger than $100 \mathrm{~km} \mathrm{~s}^{-1}$ (the value that matches the secondary, see below) to achieve a good fit of the primary's lines.

For the secondary, we have utilised the non-LTE code of Hillier \& Miller (1998) which solves the radiative transfer equation subject to the constraints of statistical and radiative equilibrium, in a spherical, extended atmosphere. Line blanketing is incorporated directly through the use of a super-level approach. We use a similar atomic model to that employed by Crowther et al. (2002) in their study of early-type O supergiants, including H I, He I-II, C III-IV, N III-V, O III-VI, Si IV, P IVv, S IV-VI and Fe IV-VII (see Crowther et al. for further details).

The velocity in the supersonic part of the wind is described by a standard $\beta$-law:

$v(r)=v_{\infty}\left(1-\frac{R_{*, \mathrm{~s}}}{r}\right)^{\beta}$

whilst the atmospheric structure, connecting the spherically extended hydrostatic layers to the $\beta$-law wind is achieved via a parameterized scale height, $h$ (see Hillier et al. 2002 for details), for which $h=0.001$ yields a reasonable match to He I and Balmer line wings, consistent with $\log g=3.5$.

The synthetic photospheric spectrum of the primary and the synthetic photospheric + wind spectrum of the secondary are combined allowing for the visual brightness ratio $\left(m_{V, \mathrm{p}}-m_{V, \mathrm{~s}}=\right.$ 0.3 ) and the parameters of the secondary model are fine-tuned to match the observed line profiles. We adjust the stellar temperature $^{1}$ and mass-loss rate of an individual model until the "photospheric" He II $\lambda 4542$ and He I $\lambda 4471$ lines are reproduced. Simultaneously, we vary the mass-loss rate until $\mathrm{H} \alpha$ is also matched. The exponent of the $\beta$-law is adjusted until the shape of $\mathrm{H} \alpha$ is well reproduced - for HDE 228766 we find $\beta \sim 1.5$. Since HDE 228766 is very heavily reddened and no UV spectra are available, we adopt a representative terminal wind velocity of $v_{\infty}=1800 \mathrm{~km} \mathrm{~s}^{-1}$. Our calculations assume a microturbulence $v_{\text {turb }}=10 \mathrm{~km} \mathrm{~s}^{-1}$ and, due to the severe blending of the lines, we nominally adopt $v \sin i=100 \mathrm{~km} \mathrm{~s}^{-1}$ for the secondary.

As discussed by Hillier et al. (2002), it is extremely difficult to determine accurate $\mathrm{He} / \mathrm{H}$ abundances in $\mathrm{O}$ supergiants. Consequently, we adopt $\mathrm{He} / \mathrm{H}=0.2$ by number, whilst $\mathrm{C}$ and $\mathrm{N}$ abundances are varied until diagnostic optical line profiles are reproduced. In Fig. 9, we present selected optical line profile fits. Overall, agreement for $T_{\mathrm{eff}, \mathrm{s}}=38000 \mathrm{~K}$ is good. He I $\lambda 4471$ provides our main temperature constraint since other blue optical He I lines are weak or absent. Alternatively, we considered using He I $\lambda 5876$ (or He I $\lambda$ 10830) together with the He II $\lambda 4686$ line. However, this method (followed by Crowther \& Bohannan 1997) yields significantly ( 4000 K) lower stellar temperatures, and suffers from inconsistencies involving the ionization balance of UV/optical metal lines.

\footnotetext{
${ }^{1}$ Defined, as is usual for an extended atmosphere, as the effective temperature corresponding to the radius at a Rosseland optical depth of 20 .
}

Table 5. Summary of the main parameters of our non-LTE analysis of the spectrum of HDE 228766.

\begin{tabular}{lcc}
\hline \hline & Primary & Secondary \\
\hline$T_{\text {eff }}(\mathrm{K})$ & 36000 & 38000 \\
$\log \left(L_{\mathrm{bol}} / L_{\odot}\right)$ & 5.58 & 5.74 \\
$R_{*}\left(R_{\odot}\right)$ & 16 & 17 \\
$\log g$ & 3.5 & \\
$\dot{M}\left(M_{\odot} \mathrm{yr}^{-1}\right)$ & & $10^{-5}$ \\
$v \sin i\left(\mathrm{~km} \mathrm{~s}^{-1}\right)$ & 200 & 100 \\
\hline
\end{tabular}

Therefore, we have greater confidence in our adopted diagnostics, which do not suffer from such problems.

A reasonable fit of the combined spectrum is achieved for $R_{*, \mathrm{~s}}=17 R_{\odot}\left(L_{\mathrm{bol}, \mathrm{s}}=550000 L_{\odot}\right)$. Assuming the secondary to solely contribute to the $\mathrm{H} \alpha$ and $\mathrm{He}$ II $\lambda 4686$ emission, its massloss rate is $10^{-5} M_{\odot} \mathrm{yr}^{-1}$. We note that our value of the mass loss rate is in perfect agreement with the estimate of MC77 who used the strength of the $\mathrm{H} \alpha$ line to infer $\dot{M}=10^{-5} M_{\odot} \mathrm{yr}^{-1}$. Moderate clumping would reduce this value by a factor of $\sim 2$.

Our primary nitrogen abundance diagnostics is

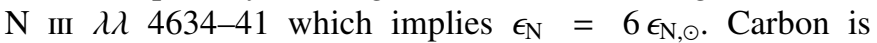
somewhat more difficult to constrain, with an upper limit to the strength of C III $\lambda \lambda 4647-51$ of $\epsilon_{\mathrm{C}}=0.08 \epsilon_{\mathrm{C}, \odot}$. As discussed elsewhere (e.g. Hillier et al. 2002), oxygen is exceedingly difficult to constrain in mid-O supergiants due to the lack of suitable optical diagnostics.

While the synthetic model fits the $\mathrm{H}_{\mathrm{I}}$ Balmer, He II (including He II $\lambda$ 10124), $\mathrm{N}$ III and $\mathrm{N}$ IV lines pretty well, the fit of the He I $\lambda 4471$ profile is less good and the model fails to reproduce the observed $\mathrm{He}$ I $\lambda \lambda$ 5876, 10830 emission profiles as well as the $\mathrm{N} v \lambda \lambda$ 4604, 4620 lines. To match the He I profiles, the temperature of the model would have to be much lower and would therefore be in disagreement with the value derived from the optical nitrogen lines. While a significant fraction of the $\mathrm{He}$ I emission could be associated with the primary wind ( $\mathrm{He}$ I $\lambda 10830$ emission is common among O supergiants, e.g. Andrillat \& Vreux 1979) or with an interaction region, our S-wave analysis clearly indicates that the $\mathrm{N} v$ lines belong to the same star (i.e. the secondary) as the $\mathrm{N}$ III and $\mathrm{N}$ iv lines. The effective temperature of the secondary indicates an 06-7 spectral type, in agreement with the results derived from the $E W$ ratio of the $\mathrm{He}$ I $\lambda 4471$ and $\mathrm{He}$ II $\lambda 4542$ lines. However, the puzzling presence of the $\mathrm{N} \vee \lambda \lambda 4604$, 4620 lines cannot be explained by this temperature. A higher temperature of approximately 40000-42000 K would be needed to fit the $\mathrm{N} v$ lines. However, the temperature cannot be much larger than $38000 \mathrm{~K}$ because otherwise the $\mathrm{N}$ iv line gets too strong.

The spectrum of HDE 228766 is remarkably similar to that of the O4If ${ }^{+}$star HDE 269698 in the LMC. HDE 269698 also displays $\mathrm{N}$ III, $\mathrm{N}$ IV and $\mathrm{N} v$ lines in its spectrum and Crowther et al. (2002) derive $T_{\text {eff }}=40000 \mathrm{~K}$ for this star, which also provides a good match to $\mathrm{He} \mathrm{I} / \mathrm{He}$ II, UV, and far-UV lines.

The structure of the secondary's atmosphere in HDE 228766 could be intermediate between the winds of HDE 269698 and of the WN7ha star in WR 22. The latter star 

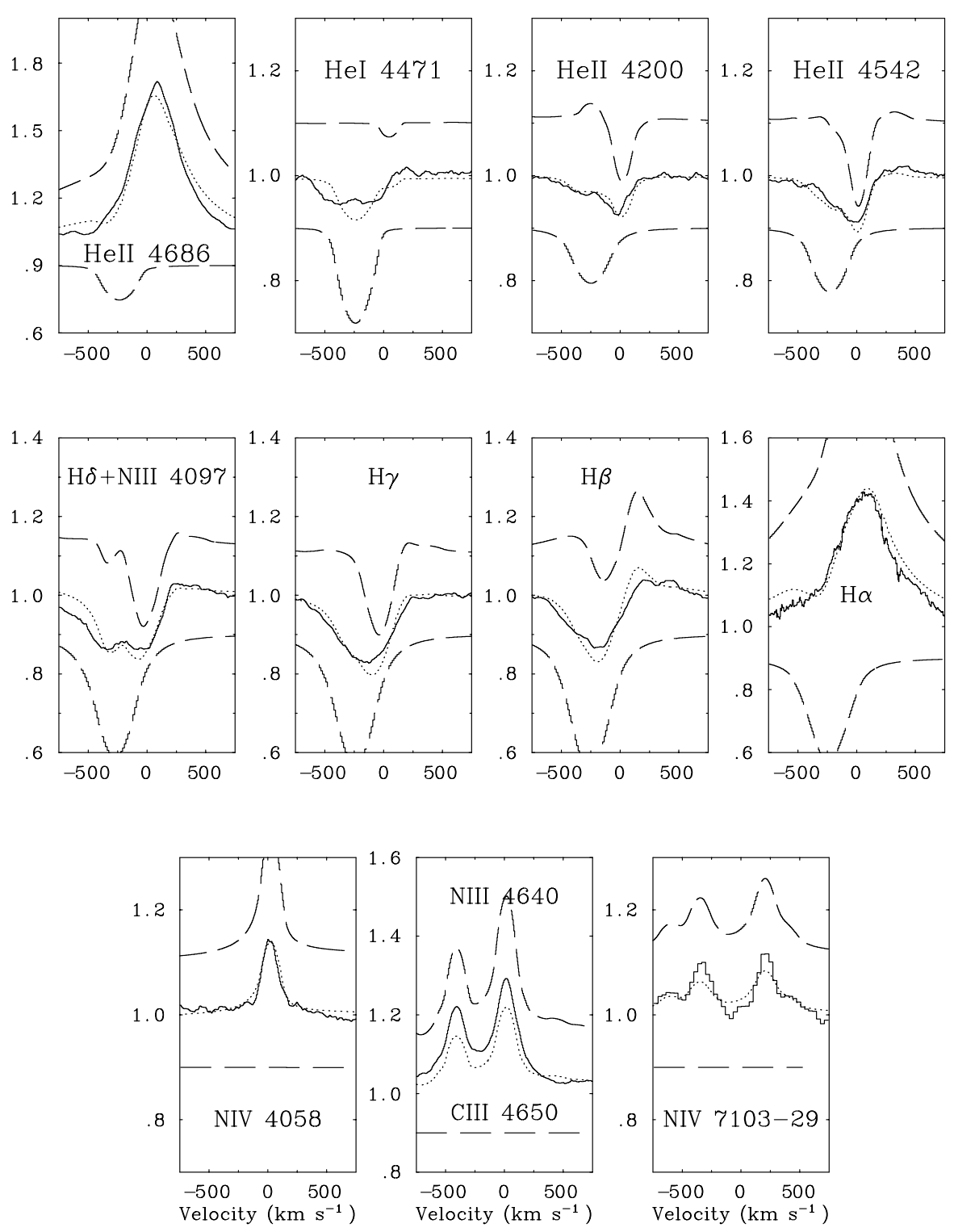

Fig. 9. Fit of the spectrum of HDE 228766 as observed in September 2000 (except for the N iv $\lambda \lambda 7103-29$ lines observed in August 1990) with a non-LTE model atmosphere code. The combined synthetic model is shown by the dotted line, whilst the observations are displayed by the continuous line. The individual normalized synthetic spectra are shown by the dashed lines offset by -0.1 and +0.1 for the primary and the secondary respectively. The synthetic spectra have been shifted in velocity to match the radial velocities of the two components at the orbital phases of the observations.

also displays $\mathrm{N}$ III, $\mathrm{N}$ IV and $\mathrm{N} v$ emission lines in its spectrum (Rauw et al. 1996). Note that an analysis of the H I, He I and He II spectrum of WR 22 with a non-LTE code yielded a rather low stellar temperature $\left(T_{*}=31900 \mathrm{~K}\right.$, Crowther et al. 1995), whereas the He II, N III and N IV lines profiles were reasonably well reproduced using a higher stellar temperature $\left(T_{*}=36500 \mathrm{~K}\right.$, Crowther et al. 1995).

Combining the results of our tomographic analysis, the mean $E W$ of the He II $\lambda 4686$ line in the observed spectrum of HDE 228766 and the optical brightness ratio we infer that the $E W$ of the intrinsic $\mathrm{He}$ II $\lambda 4686$ emission in the secondary's spectrum is of order $\sim 10 \AA$. This puts the secondary's intrinsic emission strength beyond the nominal Of range, but below that of regular WNL stars. However a WNLha classification (see Smith et al. 1996) could account for the observed line strength. In fact, comparing with the observed spectra of the presumably single WNLha stars WR 25 (WN7ha) and WR 108 (WN9ha), we find the secondary in HDE 228766 to be intermediate in excitation between these two objects, but similar in wind strength. These results suggest that a WN8ha classification might be appropriate.

\subsection{Evolutionary status}

Although the parameters derived hereabove depend on the unknown distance of HDE 228766 and the poorly constrained optical brightness ratio, our analysis clearly reveals an evolved secondary star as witnessed by the large mass loss rate and the peculiar abundances. The secondary star in HDE 228766 
appears therefore most likely as a kind of transition object at the beginning of the WR stage.

Adopting the parameters inferred hereabove, we can compare the properties of the stars in HDE 228766 with the evolutionary tracks of Schaller et al. (1992). The most stricking feature is that the secondary appears to be the most evolved component and is clearly overluminuous for its present-day mass. Hence, the secondary must have been the initially more massive component of the system. The only way to account for this situation in the framework of single star evolutionary models would be if the secondary were moving bluewards after an excursion towards the lower temperature regime. Note that in this case, the evolutionary model suggests that the secondary would be a fully developed WN Wolf-Rayet star.

Alternatively, the evolution of the whole system could have been influenced by mass exchange. Evolutionary tracks from Vanbeveren \& de Loore (1994) and de Loore \& Vanbeveren (1994) could indeed account for the overluminosity of the secondary. The initially more massive component could have undergone mass loss through Roche lobe overflow (RLOF) and would now be the less massive star. We note also that the primary has broader lines and seems to be rotating faster than the secondary. If the stars were in synchronous rotation, they should roughly have the same $v \sin i$. In the framework of a binary evolution model, the larger $v \sin i$ of the presentday primary could be due to spinning up through accretion (Vanbeveren \& de Loore 1994). It seems therefore plausible that HDE 228766 is in a post RLOF stage. The evolutionary tracks of Vanbeveren \& de Loore (1994) indicate that the secondary should be in the WNL stage.

\section{3. $A$ wind interaction in HDE 228766?}

Our data reveal a weak $\mathrm{H} \alpha$ emission feature that could be an indication of a wind interaction in the optical spectrum of HDE 228766. A colliding wind phenomenon could also produce a signature in the $\mathrm{X}$-ray domain through a phasedependent excess X-ray emission (e.g. Stevens et al. 1992).

We have therefore searched the ROSAT archive for observations of HDE 228766. Unfortunately there is only a single pointed observation (rp500248a01) where the star happened to lie within the field of view of the ROSAT-PSPC (though it was outside the inner ring of the PSPC detector). Using our new ephemeris we find that the orbital phase of HDE 228766 at the time of the PSPC observation was $\phi=0.64$. We extracted a background corrected spectrum using the XSELECT software. We have fitted this spectrum with an absorbed single-temperature optically thin plasma model (Raymond \& Smith 1977) within the XSPEC software. Fixing the neutral hydrogen column density at the interstellar value of $N_{\mathrm{H}}^{\mathrm{ISM}}=0.53 \times 10^{22} \mathrm{~cm}^{-2}$, we obtain a best fitting temperature $k T=0.88_{-.16}^{+.25} \mathrm{keV}$. The corresponding dereddened $\mathrm{X}$-ray flux in the energy band $0.1-2.0 \mathrm{keV}$ is $1.6 \times 10^{-12} \mathrm{erg} \mathrm{cm}^{-2} \mathrm{~s}^{-1}$ yielding an unabsorbed X-ray luminosity $^{2}$ of $L_{X}=2.34 \times 10^{33} \mathrm{erg} \mathrm{s}^{-1}$ for a distance of $3.5 \mathrm{kpc}$. If the total bolometric luminosity of the binary amounts to

\footnotetext{
${ }^{2}$ We caution that our fit does not account for any intrinsic - i.e. circumstellar wind - absorption.
}

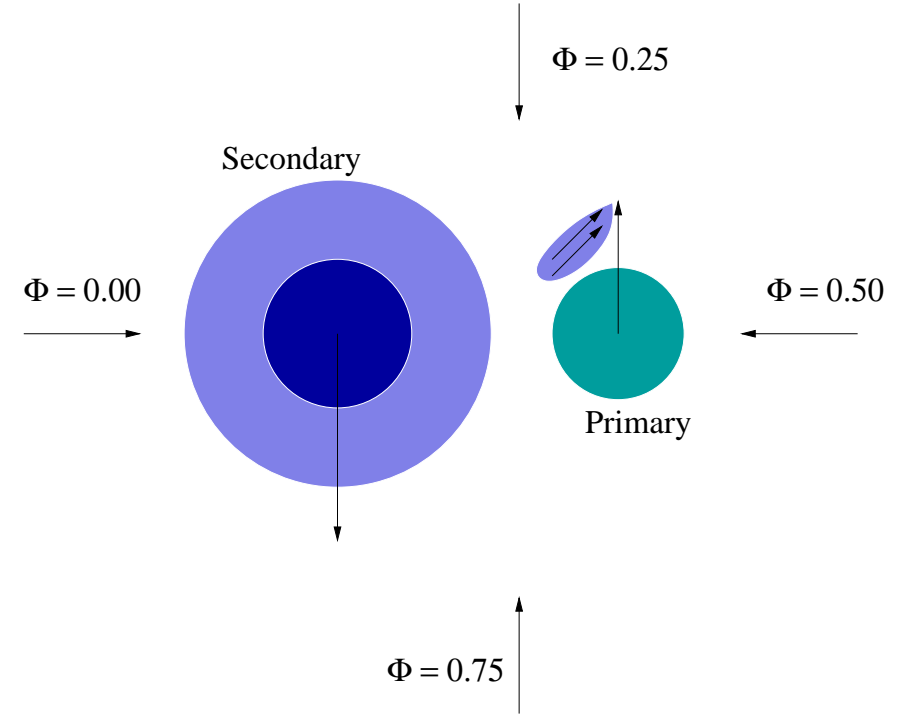

Fig. 10. Schematic view of the $\mathrm{H} \alpha$ emission region in the HDE 228766 binary system. The projection of the line of sight onto the plane of the orbit is indicated at four orbital phases. The bulk of the emission arises in the wind of the secondary star. An additional contribution comes from the small shaded area close to the primary's surface. The arrows indicate the direction of the orbital motion of the stars and of the velocity of the material in the small emitting region.

$L_{\text {bol }}=9.3 \times 10^{5} L_{\odot}($ see above $)$, we obtain $\log L_{\mathrm{X}} / L_{\mathrm{bol}}=$ -6.18 . The $L_{\mathrm{X}} / L_{\mathrm{bol}}$ ratio appears a factor $\sim 3.6$ larger than expected from the empirical relation for single O-stars given by Berghöfer et al. (1997). Although the snapshot ROSAT observation does not provide any information about the variability of the X-ray flux, it seems tempting to associate the X-ray "overluminosity" with a binary interaction.

Adopting the mass ratio $q=1.67$ derived in Sect. 4, we can estimate the minimum radii of the Roche lobes of the components using the formula of Eggleton (1983). In this way we find $R_{\mathrm{RL}} \sin i=32.2$ and $25.5 R_{\odot}$ for the primary and secondary respectively. From our non-LTE model hereabove, we infer a radius of $17 R_{\odot}$ for the secondary. Therefore it appears rather unlikely that any of the components currently fills up its critical volume. The interaction in HDE 228766 is therefore more likely associated with a wind collision rather than an ongoing Roche lobe overflow.

Since the secondary wind is by far more energetic than that of the primary, it is likely that the former crashes onto the surface of the primary star. Such a shock could in turn compress the primary wind resulting in a high density region near the point of head-on wind collision, where the weak $\mathrm{H} \alpha$ emission would be formed through recombination. Figure 10 illustrates a possible model for the location of the $\mathrm{H} \alpha$ emitting material in HDE 228766. While the bulk of the emission is produced in the stellar wind of the secondary, the weaker emission component could arise in a plasma volume which is passing by the primary's surface. This geometry could at least qualitatively account for the double-peaked line shape observed near the quadrature phases. 


\subsection{Final remarks and conclusions}

Our analysis of HDE 228766 suggests that the secondary star is the most evolved component of the system and accounts for almost all the emission features seen in the spectrum. The only exception to this rule comes from a weak $\mathrm{H} \alpha$ emission component that moves in anti-phase with the secondary and arises most probably in a wind interaction region near the surface of the primary.

HDE 228766 offers the unique opportunity to study the fundamental properties of a massive star which is just in the transition between an $\mathrm{Of}^{+}$and a WNL Wolf-Rayet star. However, a couple of crucial ingredients are missing that prevent us from deriving absolute values for some parameters. The most obvious parameter that needs to be determined is the distance. Future astrometric observations (e.g. with ESA's planned GAIA satellite) will hopefully allow to solve this problem. Moreover, a photometric monitoring of the binary over an entire orbital cycle is badly needed to clarify whether or not the system displays photometric eclipses. Our orbital solutions suggest that the orbital inclination $i$ might be large enough for such eclipses to occur. If this is the case, then an analysis of the light curve should allow to constrain not only the inclination, but also the radii of the stars and their relative brightness. This would make HDE 228766 a cornerstone for our understanding of massive star evolution.

Acknowledgements. The authors would like to thank the referee Dr. H. Henrichs for a careful reading of the manuscript. The Liège team is greatly indebted to the Fonds National de la Recherche Scientifique (Belgium) for multiple assistance including the financial support for the rent of the OHP telescope in 1999 and 2000 through contract 1.5.051.00 "Crédit aux Chercheurs" FNRS. The travels to OHP for the observing runs were supported by the Ministère de l'Enseignement Supérieur et de la Recherche de la Communauté Française. This research is also supported in part by contracts $\mathrm{P} 4 / 05$ and P5/36 "Pôle d'Attraction Interuniversitaire" (SSTC-Belgium) and through the PRODEX XMM-OM Project. PAC acknowledges financial support from the Royal Society. GR would like to thank the staff of the Observatoire de Haute Provence for their technical support during the various observing runs. The SIMBAD database has been consulted for the bibliography.

\section{References}

Andrillat, Y., \& Vreux, J.-M. 1979, A\&A, 76, 221

Berghöfer, T. W., Schmitt, J. H. M. M., Danner, R., \& Cassinelli, J. P. 1997, A\&A, 322, 167

Bohannan, B., \& Crowther, P. A. 1999, ApJ, 511, 374

Chlebowski, T., Harnden, F. R. Jr., \& Sciortino, S. 1989, ApJ, 341, 427

Conti, P. S. 1973, ApJ, 179, 181

Conti, P. S., \& Alschuler, W. R. 1971, ApJ, 170, 325

Conti, P. S., \& Howarth, I. D. 1999, MNRAS, 302, 145

Crowther, P. A., Hillier, D. J., \& Smith, L. J. 1995, A\&A, 293, 403

Crowther, P. A., \& Bohannan, B. 1996, in Wolf-Rayet Stars in the Framework of Stellar Evolution, Proc. 33rd Liège Int. Astroph. Coll., ed. J.-M. Vreux, A. Detal, D. Fraipont-Caro, E. Gosset, \& G. Rauw, 437
Crowther, P. A., \& Bohannan, B. 1997, A\&A, 317, 532

Crowther, P. A., et al. 2002, ApJ, submitted

de Loore, C., \& Vanbeveren, D. 1994, A\&A, 292, 463

Demers, S., \& Fernie, J. D. 1964, PASP, 76, 350

Eggleton, P. P. 1983, ApJ, 268, 368

Gosset, E., Royer, P., Rauw, G., Manfroid, J., \& Vreux, J.-M. 2001, MNRAS, 327, 435

Hackwell, J. A., Gehrz, R. D., \& Smith, J. R. 1974, ApJ, 192, 383

Hart, A. B. 1957, ApJ, 126, 463

Heck, A., Manfroid, J., \& Mersch, G. 1985, A\&AS, 59, 63

Hillier, D. J., \& Miller, D. L. 1998, ApJ, 496, 407

Hillier, D. J., et al. 2002, ApJ, in press

Hiltner, W. A. 1951, ApJ, 113, 317

Hiltner, W. A. 1956, ApJS, 2, 389

Hiltner, W. A., \& Schild, R. E. 1966, ApJ, 143, 770

Horn, J., Kubát, J., Harmanec, P., et al. 1996, A\&A, 309, 521

Horne, K. 1991, in Fundamental Properties of Cataclysmic Variable Stars: 12th North American Workshop on Cataclysmic Variables and Low Mass X-ray Binaries (SDSU Press), ed. A. W. Shafter, 23

Hubeny, I., \& Lanz, T. 1995, ApJ, 439, 875

Humphreys, R. M. 1978, ApJS, 38, 309

Lafler, J., \& Kinman, T. D. 1965, ApJS, 11, 216

Massey, P., \& Conti, P. S. 1977, ApJ, 218, 431

Popova, M., \& Kraicheva, Z. 1984, Astrof. Issled. Izv. Spets. Astr. Obs., 18, 64

Rauw, G., Vreux, J.-M., Gosset, E., Hutsemékers, D., Magain, P., \& Rochowicz, K. 1996, A\&A, 306, 771

Rauw, G., Vreux, J.-M., \& Bohannan, B. 1999, ApJ, 517, 416

Rauw, G., Sana, H., Vreux, J.-M., Gosset, E., \& Stevens, I. R. 2002, in Interacting Winds from Massive Stars, ASP Conf. Ser. 260, ed. A. F. J. Moffat, \& N. St.-Louis, 449

Raymond, J. C., \& Smith, B. W. 1977, ApJS, 35, 419

Roman, N. G. 1951, ApJ, 114, 492

Ruprecht, J., Balazs, B., White, R. E., \& Sharov, A. S. 1983, SvA, 27, 358

Sana, H., Rauw, G., \& Gosset, E. 2001, A\&A, 370, 121

Schaller, G., Schaerer, D., Meynet, G., \& Maeder, A. 1992, A\&AS, 96, 269

Schmidt-Kaler, T. 1982, Physical Parameters of the Stars, LandoltBörnstein Numerical Data and Functional Relationships in Science and Technology, New Series, Group VI (Springer Verlag, Berlin)

Smith, L. F., Shara, M. M., \& Moffat, A. F. J. 1996, MNRAS, 281, 163

Stevens, I. R., Blondin, J. M., \& Pollock, A. M. T. 1992, ApJ, 386, 265

Thaller, M. L., Gies, D. R., Fullerton, A. W., Kaper, L., \& Wiemker, R. 2001, ApJ, 554, 1070

Vanbeveren, D., \& de Loore, C. 1994, A\&A, 290, 129

Vreux, J.-M., \& Andrillat, Y. 1979, A\&A, 75, 93

Walborn, N. R. 1973, ApJ, 186, 611

Walborn, N. R. 2001, in Eta Carinae and Other Mysterious Stars: The hidden opportunities of emission spectroscopy, ASP Conf. Ser., 242, ed. T. R. Gull, S. Johannson, \& K. Davidson, 217

Walborn, N. R., \& Fitzpatrick, E. L. 1990, PASP, 102, 379

Walborn, N. R., Howarth, I. D., Lennon, D. J., et al. 2002, AJ, 123, 2754

Werner, K., \& Rauch, T. 2001, in Eta Carinae and Other Mysterious Stars: The hidden opportunities of emission spectroscopy, ASP Conf. Ser. 242, ed. T. R. Gull, S. Johannson, \& K. Davidson, 229 Zakirov, M. M. 1999, Astron. Lett., 25, 229 